\title{
ADAPTIVE MESH REFINEMENT IN THE DAM-BREAK PROBLEMS
}

\author{
M. L. Garcia ${ }^{1}$, T. L. Popiolek ${ }^{2}$ \\ ${ }^{1}$ Graduate Program in Computational Modeling at the Federal University of Rio Grande \\ (marianalimagarciars@gmail.com)
}

${ }^{2}$ Institute of Mathematics, Statistics and Physics at the Federal University of Rio Grande

\begin{abstract}
This study brings an adaptive mesh strategy applied to the numerical simulation of free-surface shallow water problems. In the solver the shallow water equations are integrated with the explicit two-step Taylor-Galerkin method. Equations are first discretized in time with a Taylor's series expansion and then in space using the Garlerkin technique. The finite element method with triangular unstructured meshes is used to solve the problem. An adaptive mesh strategy is added to the solver in order to obtain more precise solutions at low computational costs. The strategy consists in a mesh refinement and smoothing procedure that uses an error indicator and an adaptation criterion for the identification of the mesh elements that will be refined. The elements identified to be refined are divided in four new elements. Refinement closure is also performed to guarantee the integrity of the new mesh. In order to ensure a smooth transition among elements of different size, a smoothing procedure is applied to the mesh after its refinement. The elements to be refined are identified by error indicators that take into account the depth and velocity gradients. The adaptation criterion is defined based on these error indicators. The dam-break problem is solved with the proposed methodology and results are compared with previous published studies.
\end{abstract}

Keywords: Adaptive meshes, finite elements, shallow water, dam-break problems.

\section{INTRODUCTION}

In recent decades considerable advances benefit the applications of Computational Fluid Dynamics (CFD). Therefore several mathematical models based on the Navier-Stokes equations have been used to solve problems in the flow of compressible and incompressible fluids. Some of these models were developed for the specific purpose of simulating the flow in shallow water, characterized by their own equations derived from the Navier-Stokes equations. The shallow water equations show their application in the study of flow behavior in channels, rivers, estuaries and lagoons, encompassing all environmental and artificial coastal systems.

In this context Shallow solver is a finite element, two-dimensional program, with triangular mesh elements, which was developed to simulate the flow of incompressible fluids, 
based on the shallow water equations. This solver was developed and implemented by Martinez [5], based on the numerical model to simulate the flow of compressible fluid proposed by Vaz dos Santos [12]. Popiolek [9] added to the solver an automatic mesh adaptation to provide an improvement through the h-refinement method. Still the Shallow solver was enhanced by Garcia [4], who added an alternative subdivision of the refining elements.

The main objective of this study is to present an adaptive mesh strategy to improve the accuracy of shallow water flow simulation, integrating to the solver a refinement scheme, an adaptation criterion, and a mesh smoothing technique. This way it is possible to build fixed and dynamic meshes. In order to analyze the adaptive mesh strategy, the dam-break problem is presented and solved using the integrated shallow water equations with the explicit twostep Taylor-Galerkin method.

Section 2 describes the procedures for the mesh adaptation strategy with error indicators, criteria for mesh adaptation, subdivision of elements, and nodal re-allocation. Section 3 presents the shallow water equations. Section 4 shows the dam-break flow simulation. Conclusions are presented in Section 5.

\section{MESH ADAPTATION}

The main objective of the adaptation is to provide a dynamic construction of optimized mesh regarding flow characteristics, in order to improve quality of the numerical solution with a low computational cost [10]. The main tools of the mesh adaptation include error indicators, an adaptation mesh criterion, a refinement scheme and smoothing process.

In mesh adaptation, error indicators are used for mapping the numerical solution behavior and identifying regions of the computational domain where an adaptive scheme is necessary to obtain an accurate numerical solution. Herein, these error indicators take into account velocity gradients and depth gradients. The error indicator is analyzed by an adaptation criterion to identify elements that will be refined in a selective way. In the refinement scheme, elements identified to be refined by the criterion adaptation are divided into four new elements, defined as a regular refinement. Irregular refinements are performed for elements with one and two divided edges. In order to improve the geometrical quality of the mesh elements and to smooth the transition among refined and non-refined elements, a smart Laplacian smoothing technique is applied.

\subsection{Errors Indicators}

Obtaining an accurate solution advocates the use of effective error indicators, capable of pointing out clearly the regions of the mesh with low precision solution and applying a mesh refinement process to them.

Error indicators are used depending on the main physical phenomena occurring in flows in shallow water bodies, taking into account the gradients of velocity and depth gradients. Further information can be found in [2]. 
The error indicator which expresses the velocity gradients in the $x$ direction may be defined by:

$$
\theta_{i}^{2}=\left\{\left|\frac{\partial u}{\partial x}\right|+\left|\frac{\partial v}{\partial x}\right|\right\} \bar{l}_{i}^{2}, i=1,2,3, \ldots, \text { nel }
$$

where $i$ represents the element number, $x$ is the $V$ gradient direction, $u$ and $v$ are respectively the components of velocity vector in the directions $x$ and $y$, nel is the total number of mesh elements, and $\overline{l_{i}}$ is the arithmetic mean of the length of the sides of the $i$-th element.

The error indicator which quantifies the gradients of water depth is defined by the following expression:

$$
\theta_{i}^{2}=\left\{\left|\frac{\partial h}{\partial x}\right|+\left|\frac{\partial h}{\partial y}\right|\right\} \bar{l}_{i}^{2}, i=1,2,3, \ldots, \text { nel },
$$

where $h$ is the water depth and $x$ and $y$ are the principal directions.

Aimed at identifying the main characteristics of the simulated flow and enabling its effects to be represented in the numerical solution, the two error indicators defined above are used in the application.

\subsection{Mesh Adaptation Criterion}

For an efficient mesh adaptation it is essential that the values of error indicators are reviewed by an appropriate criterion. In the literature several mesh adaptation criteria have been made based on the error equidistribution, such as those of [6] and [2].

This study adopted the error equidistribution methodology based on criteria that calculate the mean value of the error indicators $\left(\theta_{m}\right)$ and allow all elements of the mesh showing an error indicator $\left(\theta_{i}\right)$ greater than $\alpha \theta_{m}$ to be identified and refined. The variable $\alpha$ is an arbitrary parameter used for refinement control.

\subsection{Subdivision of Elements}

The subdivision of elements is the main phase of a mesh adaptation process. Special care is needed to provide the construction of a consistent mesh, with geometric elements of good quality.

In regular refinement the triangular element is subdivided into four new elements, called 4T-SS partition (four-triangle self-similar) [7]. In 4T-SS partition the new sides of the element are defined by straight line segments joining the midpoints of each side of the element, as shown in Figure 1.

According to Padron [7], 4T-SS subdivision is the most used in adaptations of located meshes because of its feature of generating elements similar to the refined one, thus preserving the mesh quality. 


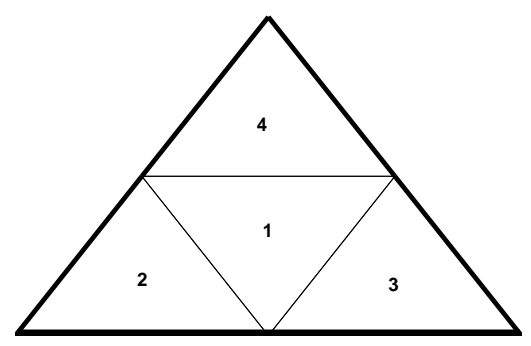

Figure 1. Subdivision into four new elements.

In carrying out the mesh closure, three situations may occur with the elements adjacent to the regular refinements, which generate irregular refinements. The first situation is when the element is adjacent to a single refined element, in which it is subdivided into two new elements to construct a consistent mesh [11] (Figure 2). The second situation occurs when the element is adjacent to two refined elements, in which it is subdivided into three new elements [8] (Figure 3). The third situation is when the element is adjacent to three refined elements, in which it is subdivided into four new element elements [3] (Figure 4).
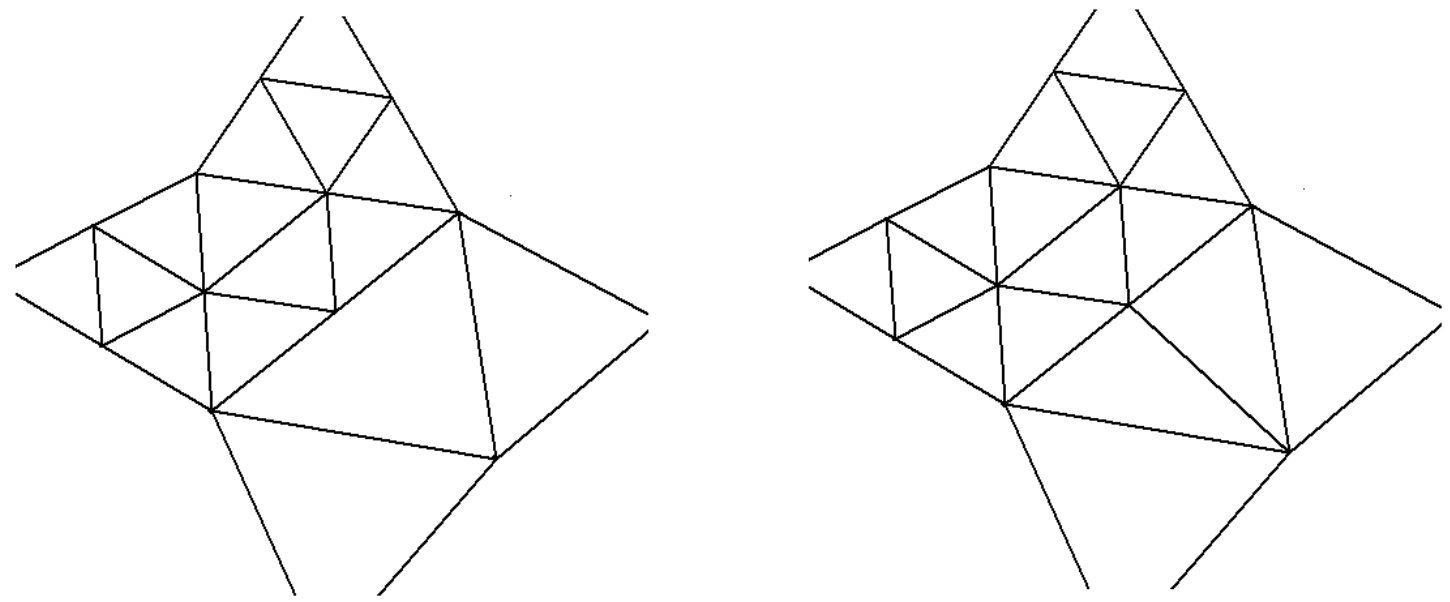

Figure 2. Refinement of the element adjacent to one refinement.
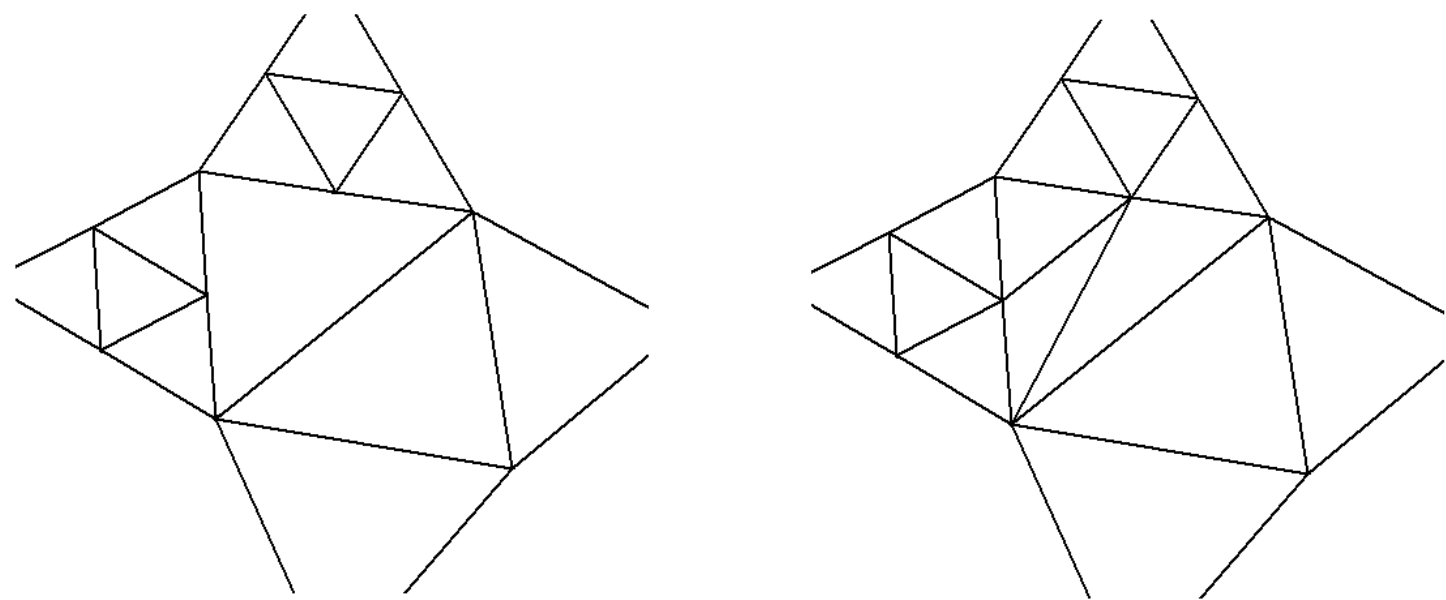

Figure 3. Refinement of the element adjacent to two refinements. 

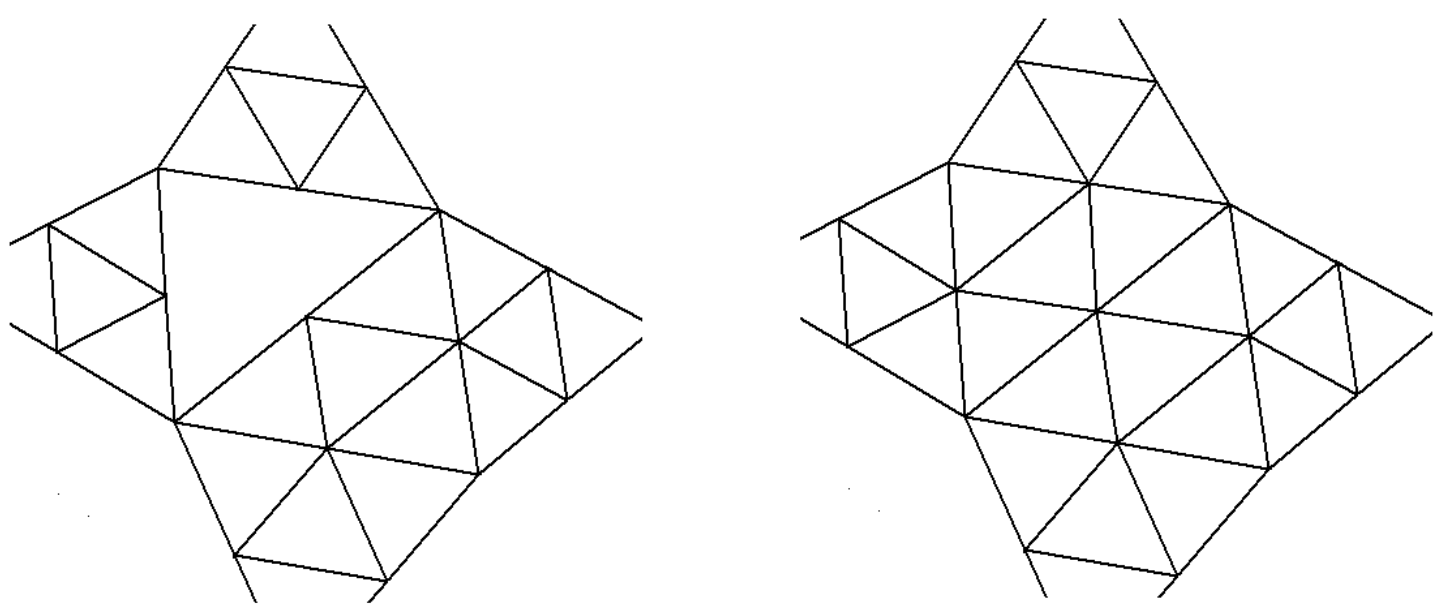

Figure 4. Refinement of the element adjacent to three refinements.

\subsection{Nodal re-allocation}

In order to improve the geometric quality of the elements in the finite element mesh and to smooth the transition among elements of different size, a smart Laplacian smoothing technique is used after each refinement.

Special care must be taken with nodes belonging to external or internal boundary when the smoothing process is applied. Some nodes remain fixed and others may have restrained displacements, remaining on the boundary. Information referred to nodes that can be re-allocated or those that cannot are given in the solver data structure, where the status of each node is defined.

\section{SHALLOW WATER EQUATIONS}

For analyzing the efficiency of the mesh adaptation scheme, procedures were incorporated into the Shallow solver as developed by Vaz dos Santos [12] to simulate flows in shallow water bodies.

The shallow water equations of the mathematical model are obtained through integration in the depth of equations of mass conservation and momentum, derived from the NavierStokes three-dimensional equations. The shallow water equations are integrated using the two-step Taylor-Galerkin explicit method.

The method is conducted in two steps using the shallow water equation in a compact form as:

$$
\frac{\partial U}{\partial t}+\frac{\partial F_{i}}{\partial x_{i}}=R_{s}+\frac{\partial R_{d i}}{\partial x_{i}}, i=1,2
$$

where $U$ is the vector of independent variables, $F_{i}$ is the convective flow vector in the direction of $i$ spatial coordinate, $R_{s}$ is the vector containing the source terms, and $R_{d i}$ is the vector 
containing the diffusive terms in the direction of $i$ spatial coordinate. Each of these vectors is defined by:

$$
U=\left[\begin{array}{c}
h \\
h U_{1} \\
h U_{2}
\end{array}\right], \quad F_{1}=\left[\begin{array}{c}
h U_{1} \\
h U_{1} U_{1}+\frac{1}{2} g\left(h^{2}-H^{2}\right) \\
h U_{1} U_{2}
\end{array}\right], \quad F_{2}=\left[\begin{array}{c}
h U_{2} \\
h U_{1} U_{2} \\
h U_{2} U_{2}+\frac{1}{2} g\left(h^{2}-H^{2}\right)
\end{array}\right],
$$

$$
R_{S}=\left[\begin{array}{c}
0 \\
\hat{f} h U_{2}+g(h-H) \frac{\partial H}{\partial x_{1}}+\frac{\tau_{31}^{S}}{\rho}-\frac{g|U| U_{1}}{C^{2}}-\frac{h}{\rho} \frac{\partial p_{a}}{\partial x_{1}} \\
-\hat{f} h U_{1}+g(h-H) \frac{\partial H}{\partial x_{2}}+\frac{\tau_{32}^{S}}{\rho}-\frac{g \mid U_{2}}{C^{2}}-\frac{h}{\rho} \frac{\partial p_{a}}{\partial x_{2}}
\end{array}\right],
$$

$$
R_{d 1}=\left[\begin{array}{c}
0 \\
-\frac{2}{3} \mu_{H} \frac{h}{\rho} \frac{\partial U_{1}}{\partial x_{1}} \\
\mu_{H} \frac{h}{\rho}\left(\frac{\partial U_{2}}{\partial x_{1}}+\frac{\partial U_{1}}{\partial x_{2}}\right)
\end{array}\right] \quad \text { e } \quad R_{d 2}=\left[\begin{array}{c}
0 \\
\mu_{H} \frac{h}{\rho}\left(\frac{\partial U_{2}}{\partial x_{1}}+\frac{\partial U_{1}}{\partial x_{2}}\right) \\
-\frac{2}{3} \mu_{H} \frac{h}{\rho} \frac{\partial U_{2}}{\partial x_{2}}
\end{array}\right]
$$

where, $h$ and $H$ are respectively the total depth and the depth of water at rest, $U_{1}$ and $U_{2}$ are components of the mean velocity which over this study are called $u$ and $v,|U|$ is the resulting mean velocity which over this study is called $\bar{V}, \tau_{31}^{S}$ and $\tau_{32}^{S}$ are the water surface shear stress, $g$ is the gravity acceleration, $\rho$ is the density, $C$ is the Chezy coefficient, $\mu_{H}$ is the eddy viscosity, $p_{a}$ is the atmospheric pressure, and $\hat{f}$ is the Coriolis coefficient.

\section{DAM-BREAK PROBLEM}

In order to validate the mesh adaptation scheme incorporated to Shallow solver, the flow from a partial dam-break was simulated and the wave propagation was studied. The geometry of the computational domain of the problem is a square measuring $200 \mathrm{~m}$ in width and $200 \mathrm{~m}$ in length, and a $75 \mathrm{~m}$ wide breach, as shown in Figure 5.

It is an inviscid flow. As shown in Figure 6, the initial velocities are zero and the initial water depths are given by:

$$
\begin{cases}h(x, y)=10 m & 0 \leq x \leq 100 \\ h(x, y)=5 m & 100<x \leq 200\end{cases}
$$




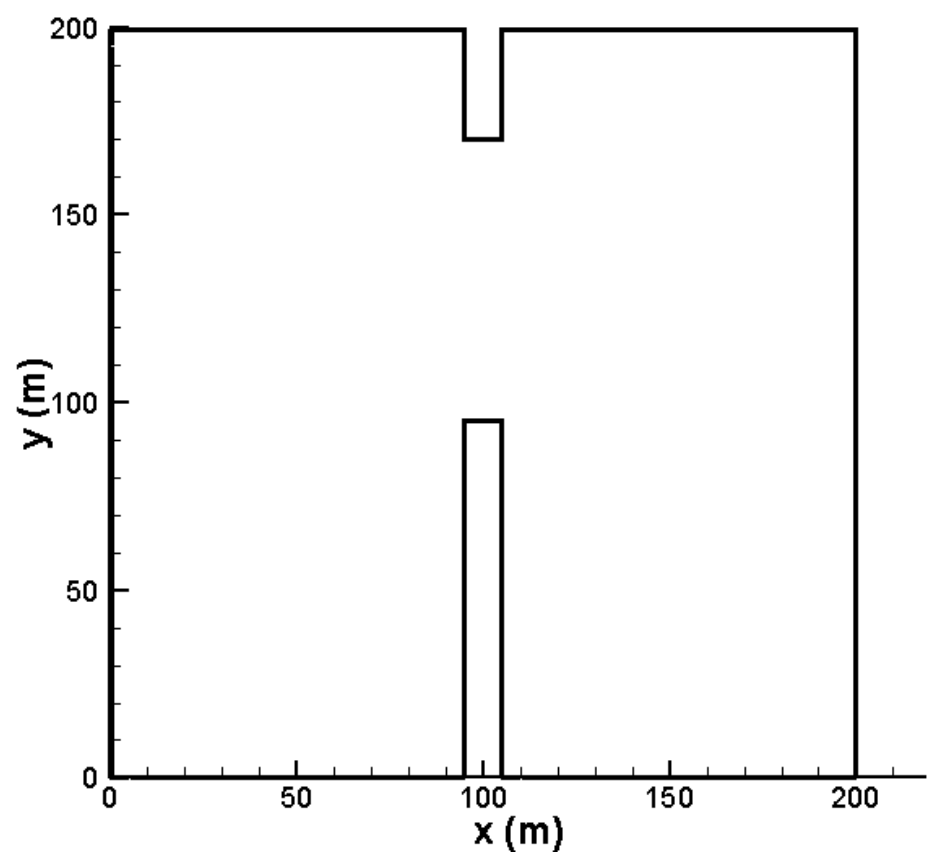

Figure 5. Computational domain of the problem.

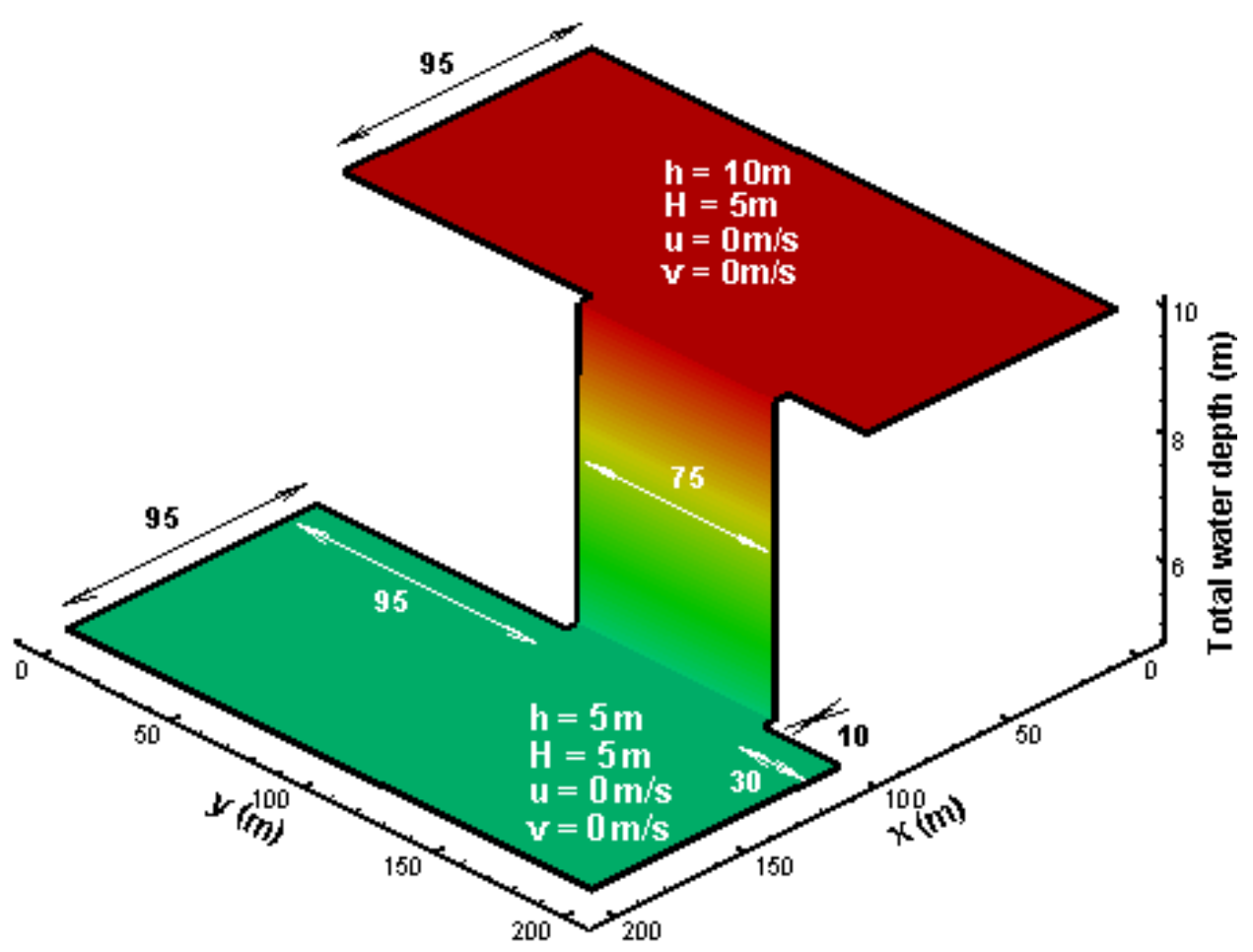

Figure 6. Initial conditions of the problem.

Initially the computational domain was discretized with a non-structured mesh containing 1847 nodes and 3482 elements. However, the numerical simulation was obtained through a mesh adapted with three levels of smoothed refinements. Error indicators were used for identifying the elements to be refined, as shown in Section 2.1, i.e. the velocity gradients in the $x$ direction (Equation 1) and the water depth gradients (Equation 2). For the mesh adap- 
tation criterion, the $\alpha$ values used were 0.6 and 0.1 respectively for the abovementioned error indicators.

Figure 7 shows the mesh adapted with three levels of refinement smoothed, containing 65548 nodes and 130418 elements, which was used for obtaining the numerical solution of the problem. It may be noticed that the refinement scheme used provided the mesh with good geometrical quality, smoothed, with no domino effect refinements and with a smooth transition among different-size elements.
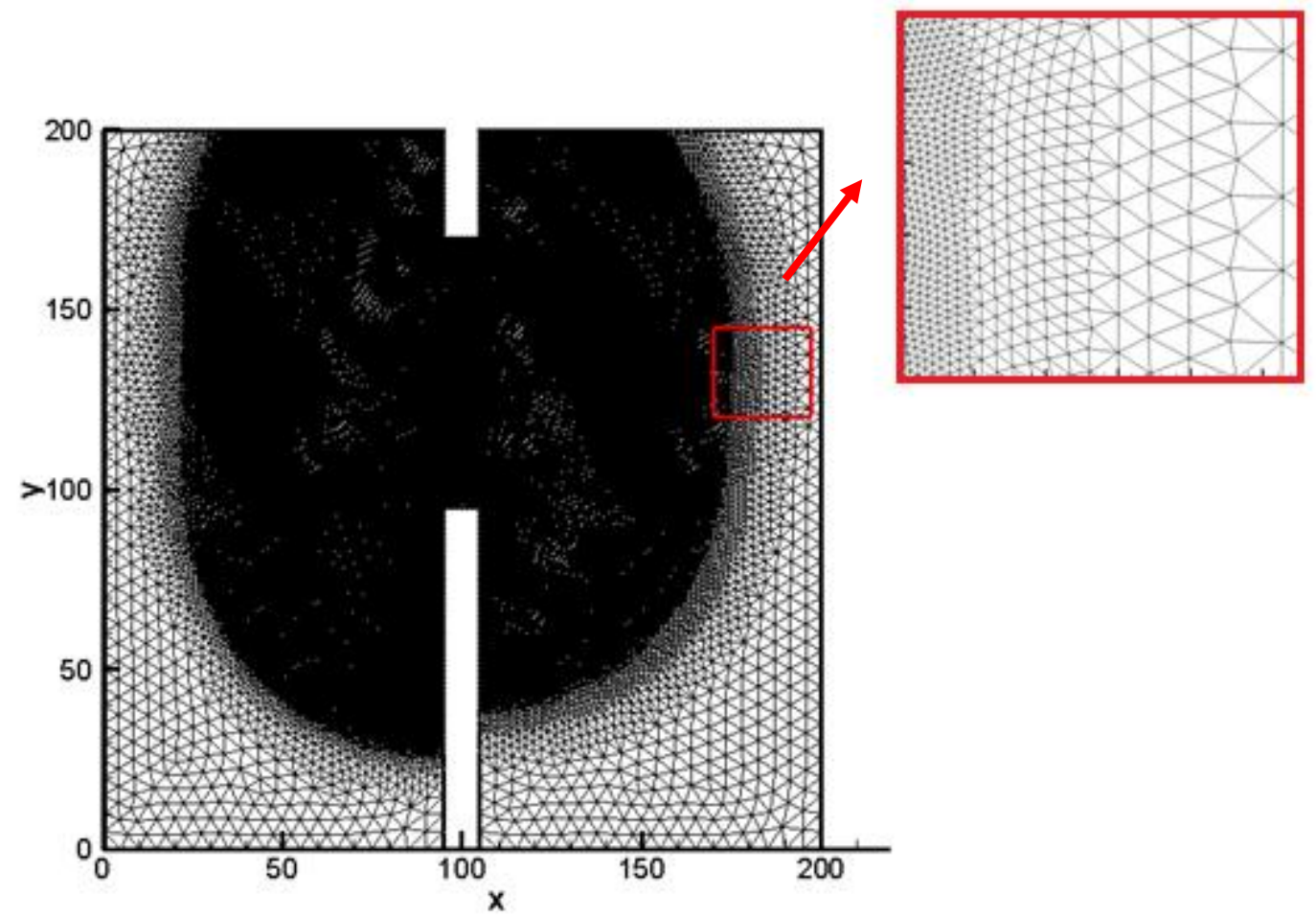

Figure 7. Refined mesh with 65548 nodes and 130418 elements.

Figure 8 shows in 3D the fields of total water depth at the times $1.2 \mathrm{~s}, 2.4 \mathrm{~s}, 3.6 \mathrm{~s}, 4.8 \mathrm{~s}$, $6.0 \mathrm{~s}$, and $7.2 \mathrm{~s}$ after the dam break.

Figure 9 shows the results of total water depth and velocity in the transversal section located along $x=92.5 \mathrm{~m}$ and at the time $7.2 \mathrm{~s}$ after the dam break. It also has the results obtained by Akoh et al. [1], which show good compliance with the ones found in this study.

Figure 10 shows the results of the profile of velocities and total water depths at the times $1.2 \mathrm{~s}, 2.4 \mathrm{~s}, 3.6 \mathrm{~s}, 4.8 \mathrm{~s}, 6.0 \mathrm{~s}$, and $7.2 \mathrm{~s}$ in the transversal section, located at $y=132.5 \mathrm{~m}$, in the middle of the breach. The charts show the variation in the profiles of velocities and total water depths over time after the dam break. 


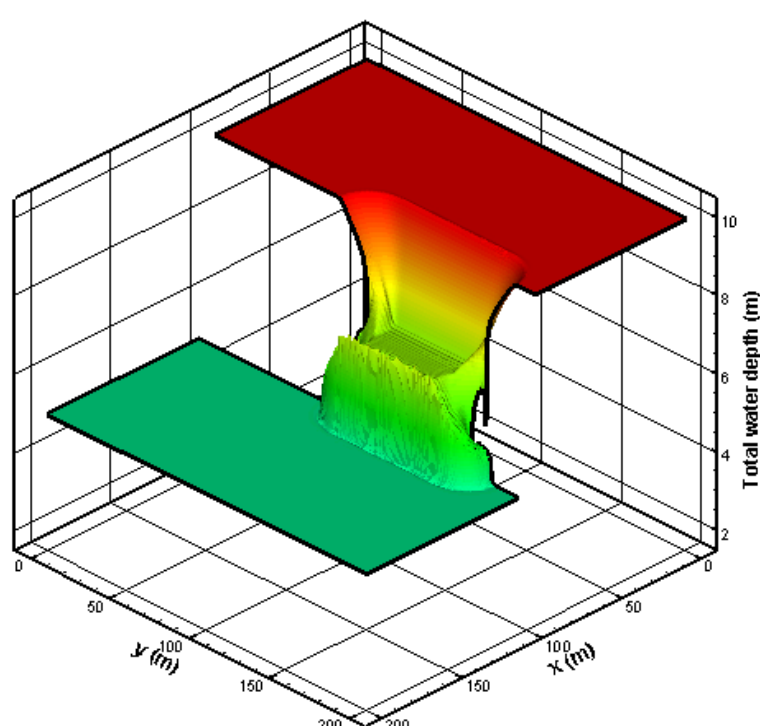

(a)

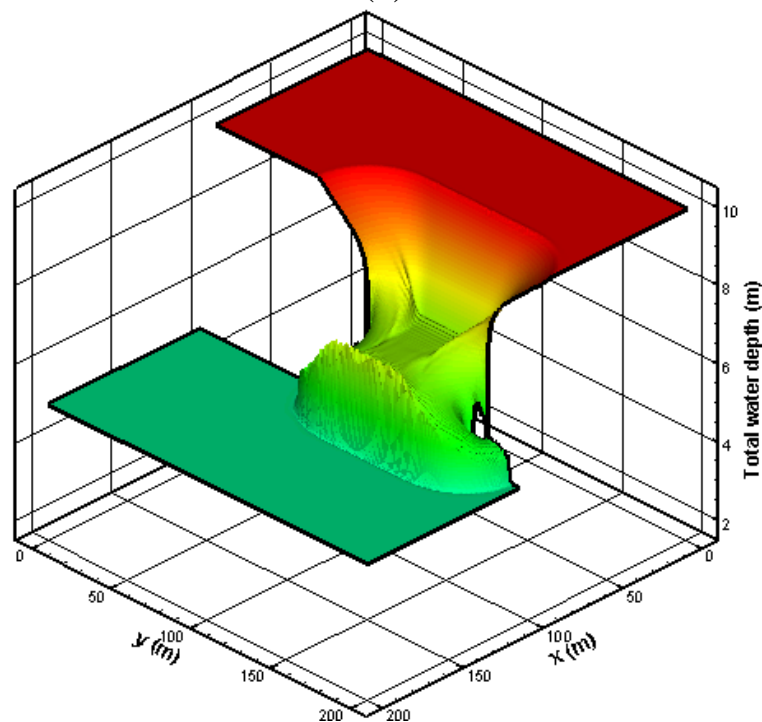

(c)

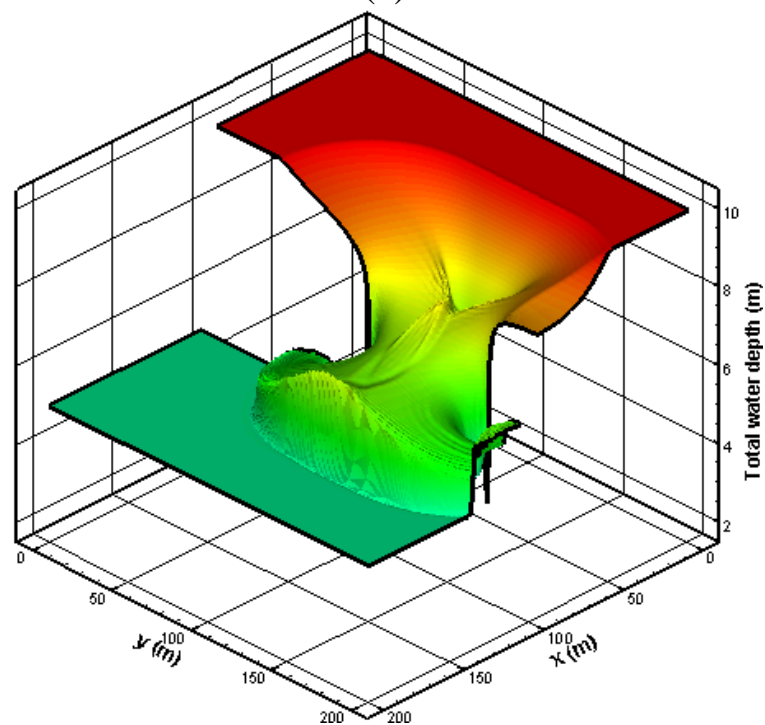

(e)

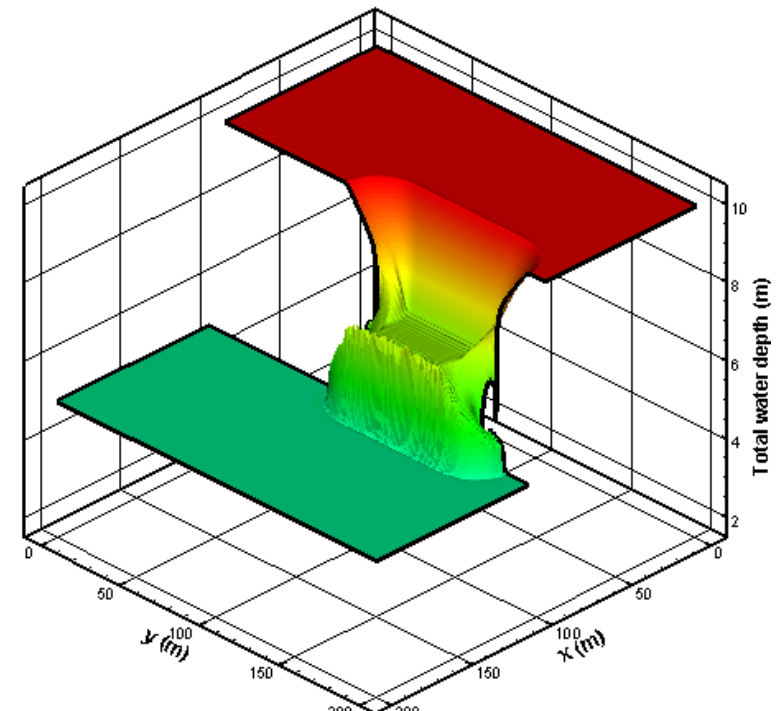

(b)

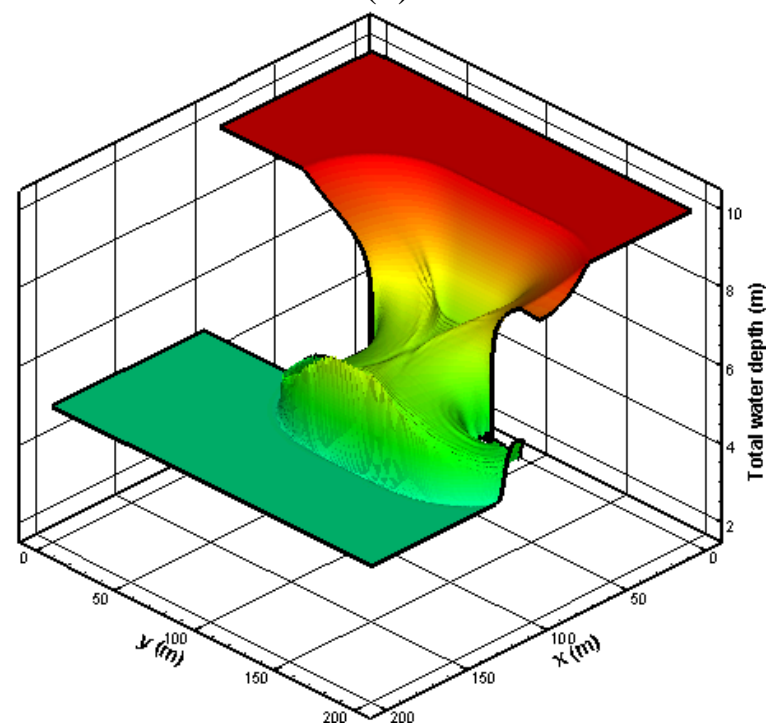

(d)

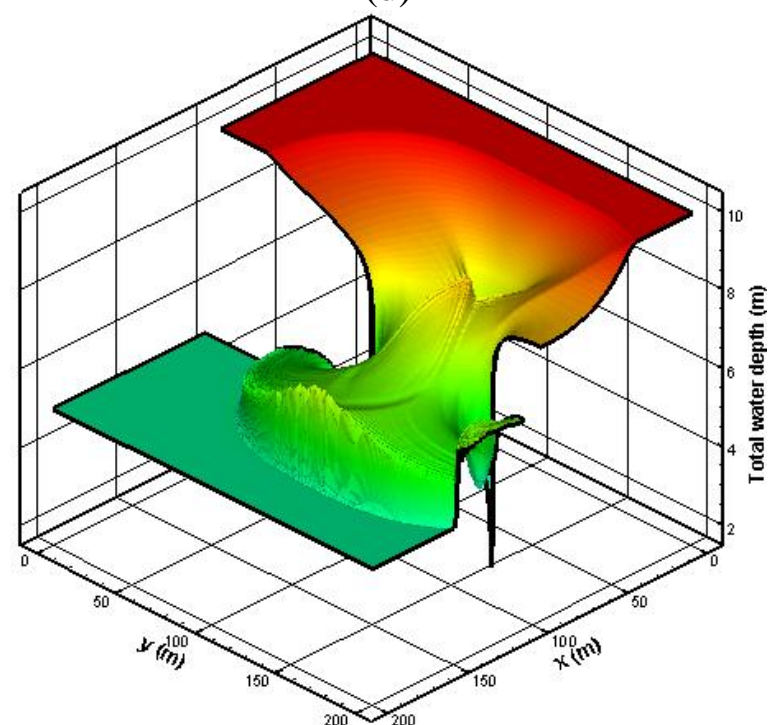

(f)

Figure 8. Fields of total water depth after the dam break: (a) $1.2 \mathrm{~s}$; (b) $2.4 \mathrm{~s}$;

(c) $3.6 \mathrm{~s}$; (d) $4.8 \mathrm{~s}$; (e) $6.0 \mathrm{~s}$; (f) 7.2s. 


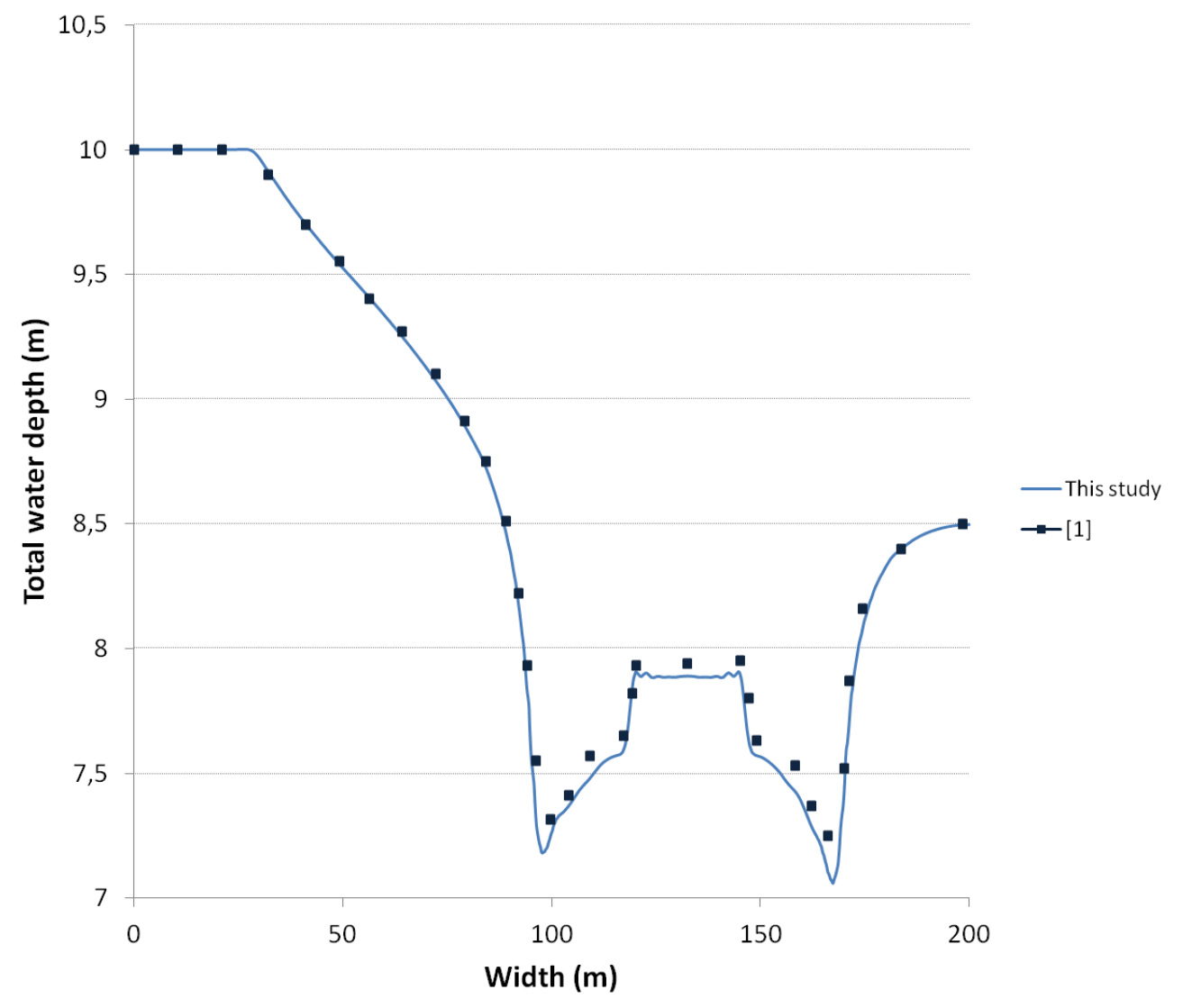

(a)

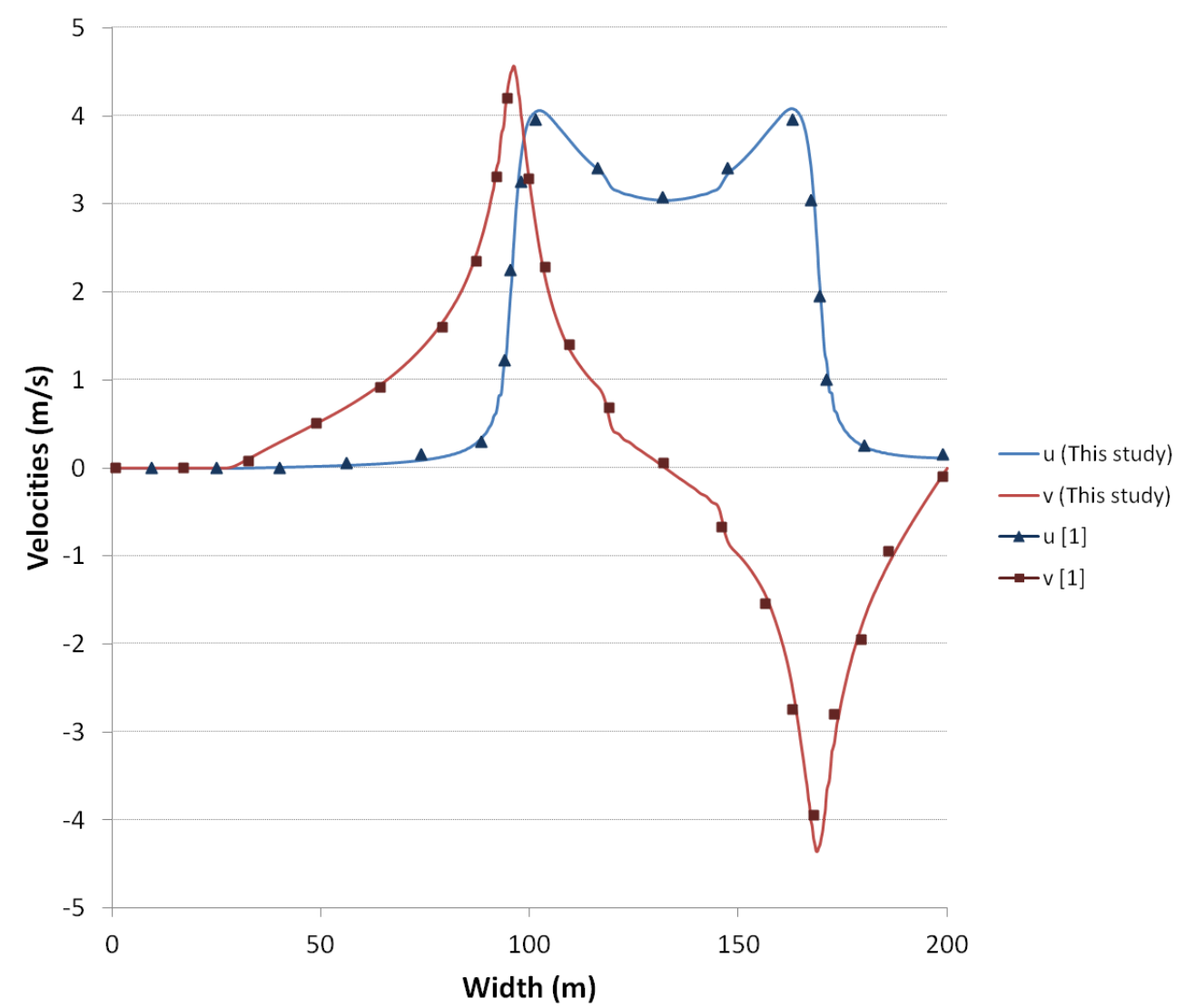

(b)

Figure 9. Comparison of results: (a) total water depths; (b) velocities. 


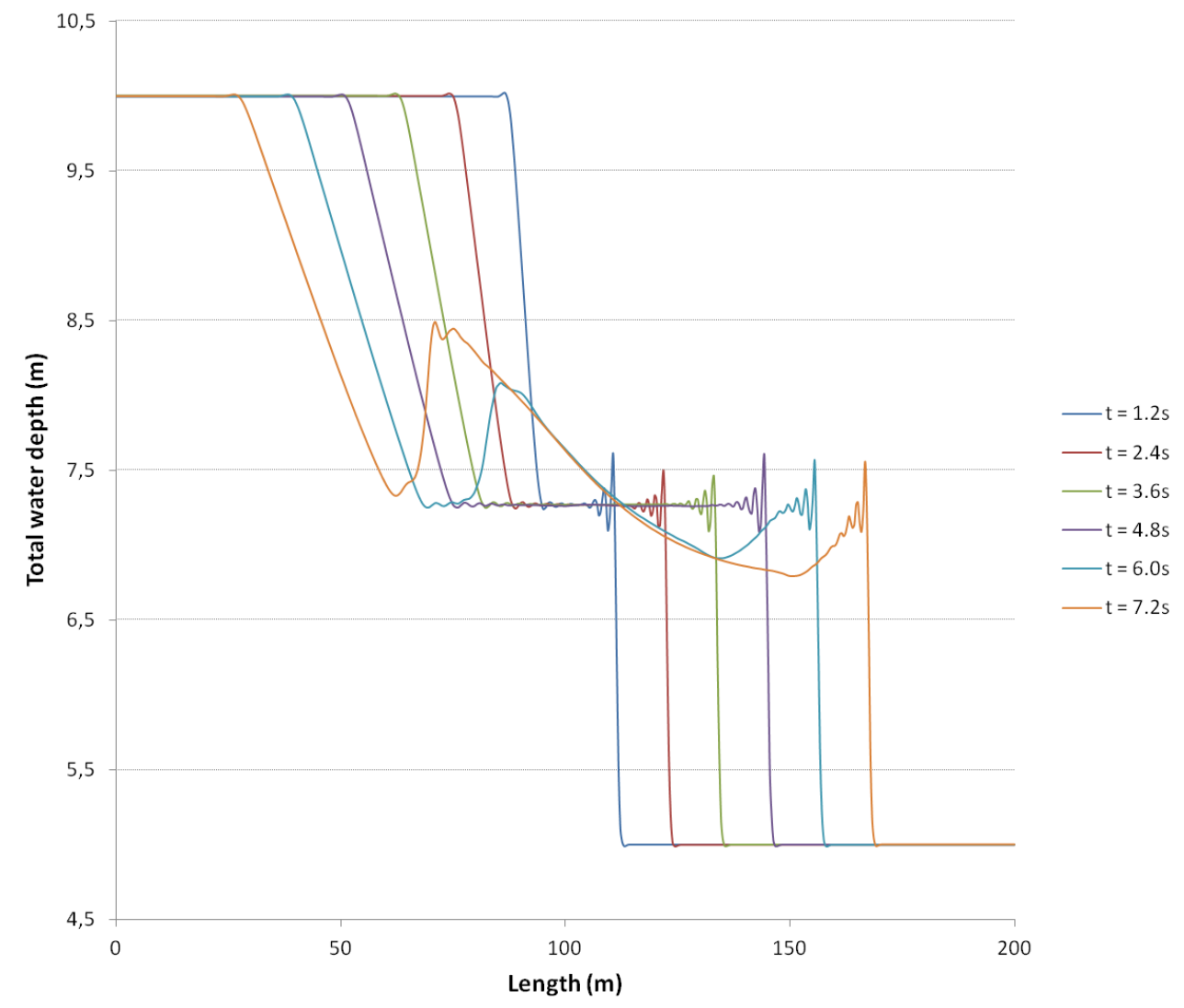

(a)

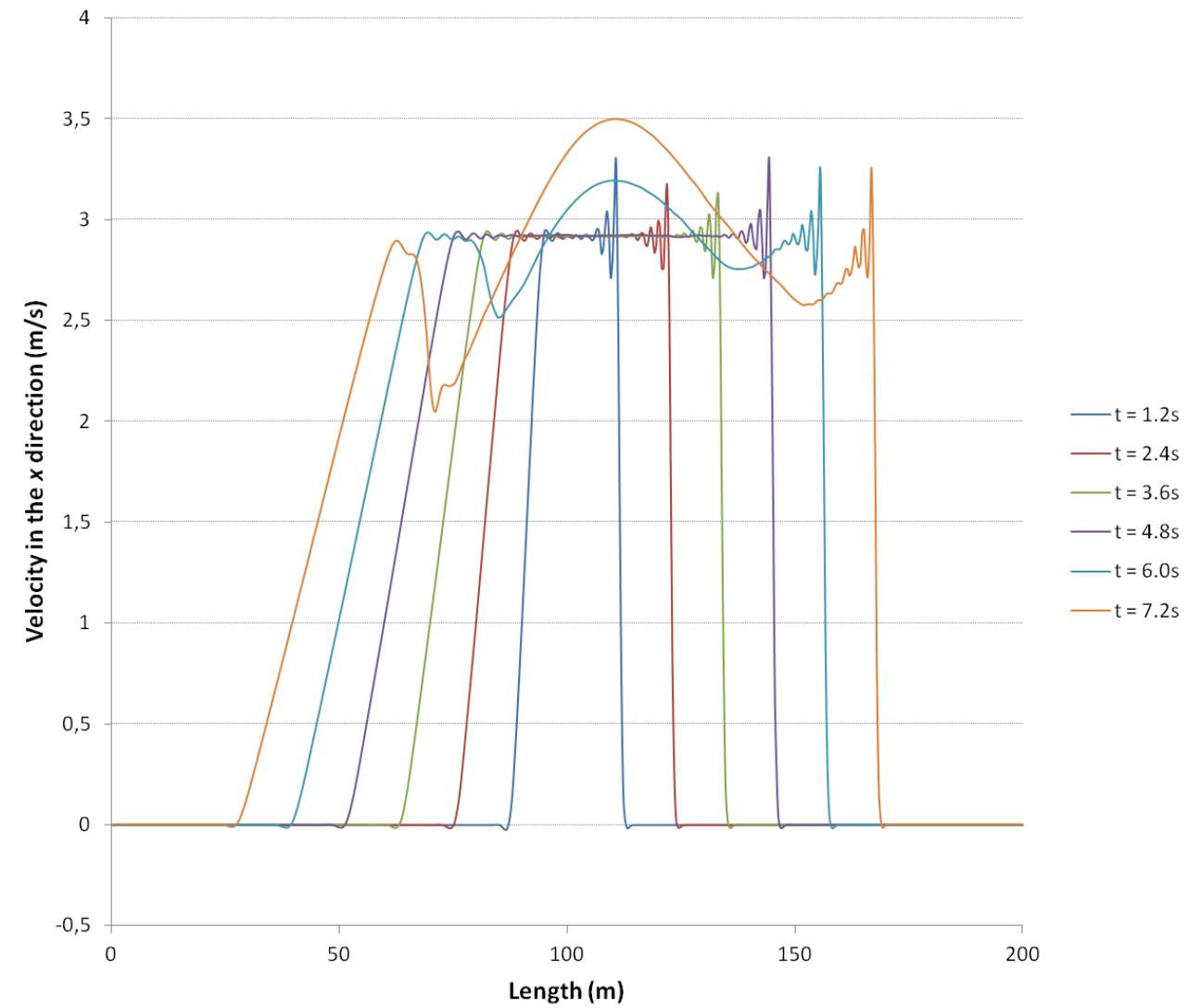

(b)

Figure 10. Results in the transversal section located along $y=132.5 \mathrm{~m}$ : (a) total water depth; (b) velocity in the $x$ direction. 


\section{CONCLUSION}

This study showed results related to a dam break. They were obtained through an element subdivision alternative of mesh adaptation strategy used for simulating the flow of freesurface shallow water.

Adaptation criterion, error indicators, refinement schemes and smoothing process were found to be effective for mesh adaptation, since the refinements were conducted in the areas of critical wave flow. Elements of geometrical quality were also generated with no domino effect and with a smooth transition among elements of different size.

Once the mesh was refined, in the areas of wave advance, because of the dam break, it was possible to obtain good-precision solution at low computational cost if compared to a refined mesh in the whole domain.

The numerical solution of this study was verified to show agreement with the results found in the literature through analysis of the results obtained at the time $7.2 \mathrm{~s}$ in the transversal section located along $x=92.5 \mathrm{~m}$.

\section{REFERENCES}

[1] Akoh R., LI S., Xiao F., "A multi-moment finite volume formulation for shallow water equations on unstructured mesh". Journal of Computational Physics. 229, 4567-4590, 2010.

[2] Argyris J., Doltsinis I. S., Friz H., "Studies on computational reentry aerodynamics". Computer Methods in Applied Mechanics and Engineering. 81, 257-289, 1990.

[3] Bank R. E., Sherman A. H., Weiser A., "Some refinement algorithms and data structures for regular local mesh refinement”. Scienitific Computing. 3-17, 1983.

[4] Garcia M. L., "Malhas Adaptativas na Simulação de Escoamentos em águas Rasas". Master dissertation PPGMC/FURG, 2011.

[5] Martinez D. M. V., "Formulação Geral Explícita para Solução de Escoamentos em Águas Rasas". Master dissertation PPGEO/FURG, 1997.

[6] Oden J. T., Strouboulis T., Devloo P., “Adaptive finite element methods for the analysis of inviscid compressible flows: Part I - Fast refinement/unrefinement and moving mesh methods for unstructured meshes". Computer Methods in Applied Mechanics and Engineering. 59, 327-362, 1986.

[7] Padrón M. A., Suárez J. P., Plaza A., "Refinement based on longest-edge and self-similar four-triangle partitions”. Mathematics and Computers in Simulation. 75, 251-262, 2007.

[8] Plaza A., Suárez J. P., Padrón M. A., "Non-equivalent partitions of d-triangles with Steiner points". Applied Numerical Mathematics. 49, 415-430, 2004. 
[9] Popiolek T. L., “Adaptação Automática de Malhas Não Estruturadas no Estudo de Escoamentos em Águas Rasas". Master dissertation PPGEO/FURG, 1999.

[10] Popiolek T. L., Awruch A. M., "Numerical Simulation of Incompressible Flows Using Adaptive Unstructured Meshes and the Pseudo-Compressibility Hypothesis". Advances in Enginee-ring Software. 37, 260-274, 2006.

[11] Rivara M. C., "Mesh refinement processes based on the generalized bisection of simplices”. SIAM Journal on Numerical Analysis. 21, 604-613, 1984.

[12] Vaz dos Santos M. A., "Simulação Numérica de escoamento compressíveis e sua interação com estruturas deformáveis”. Doctorate thesis PPGEC/UFRGS, 1993. 